DOI

\title{
КЛИНИКО-ПАТОГЕНЕТИЧЕСКОЕ ЗНАЧЕНИЕ БЕЛКА ТЕПЛОВОГО ШОКА НSР70 ПРИ ИНФАРКТЕ МИОКАРДА НА ФОНЕ ХРОНИЧЕСКОЙ ОБСТРУКТИВНОЙ БОЛЕЗНИ ЛЕГКИХ
}

\section{CLINICAL AND PATHOGENETIC 3 APPLICATION OF THE HSP70 HEAT SHOCK PROTEIN IN MYOCARDIAL INFARCTION ON THE BACKGROUND OF CHRONIC OBSTRUCTIVE LUNG DISEASE}

\author{
А.В. Наумов ${ }^{1}$, Т.В. Прокофьева ${ }^{1}$, О.С. Полунина ${ }^{1}$, Л.В. Сароянц ${ }^{2}$ \\ A.V. Naumov ${ }^{1}$, T.V. Prokofieva ${ }^{1}$, O.S. Polunina ${ }^{1}$, L.V. Saroyants ${ }^{2}$ \\ ${ }^{1}$ Астраханский государственный медицинский университет, \\ Россия, 414000, г. Астрахань, ул. Бакинская, 121 \\ ${ }^{2}$ ФГБУ «Научно-исследовательский институт по изучению лепры» Минздрава России Россия, \\ 414000, г. Астрахань, проезд Н. Островского, д. 3 \\ ${ }^{1}$ Astrakhan State Medical University, 121 Bakinskaya St., Astrakhan, 414000, Russia \\ ${ }^{2}$ «Leprosy Research Institute» Ministry of Healthcare of the Russian Federation, \\ 3 N. Ostrovsky pr., Astrakhan, 414057, Russia \\ E-mail: prokofeva-73@inbox.ru
}

\begin{abstract}
Аннотация
С целью оценки клинико-патогенетического значения белка теплового шока HSP70 у больных инфарктом миокарда (ИМ) на фоне хронической обструктивной болезни легких (ХОБЛ) обследовано 65 пациентов: 28 больных ИМ и 37 больных ИМ на фоне ХОБЛ, а также 20 соматически здоровых лиц. Уровень HSP70 определялся методом ИФА. Наибольший уровень HSP70 выявлен у больных ИМ на фоне ХОБЛ, особенно на ранних сроках госпитализации. Полученные данные о взаимосвязи HSP70 с глубиной поражения миокарда позволяют предположить возможность использования данного белка теплового шока не только как маркера ранней диагностики ИМ у пациентов с ХОБЛ, а также в качестве маркера тяжести течения ИМ у коморбидных пациентов с ХОБЛ. Это будет способствовать раннему выявлению коронарных событий у больных с ХОБЛ, что особенно важно с учетом высокой частоты стертых форм ИМ и атипичной симптоматикой ИМ у больных ХОБЛ.
\end{abstract}

\begin{abstract}
In order to assess the clinical and pathogenetic value of the heat shock protein HSP 70 in patients with myocardial infarction (MI) on the background of chronic obstructive pulmonary disease (COPD), 65 patients were examined: $28 \mathrm{MI}$ patients and $37 \mathrm{MI}$ patients with COPD, as well as 20 somatically healthy individuals. ELISA determined the level of HSP70. The highest level of HSP70 was found in MI patients with COPD, especially in the early stages of hospitalization. The obtained data on the relationship of HSP70 with the depth of myocardial lesion suggest the possibility of using this heat shock protein not only as a marker of early diagnosis of MI in patients with COPD, but also as a marker of the severity of MI in comorbid patients with COPD. This will facilitate early detection of coronary events in patients with COPD, which is especially important given the high frequency of erased forms of MI and atypical symptoms of MI in patients with COPD.
\end{abstract}

Ключевые слова: белки теплового шока, HSP70, инфаркт миокарда, хроническая обструктивная болезнь легких, коморбидность.

Keywords: heat shock proteins, HSP70, myocardial infarction, chronic obstructive pulmonary disease, comorbidity. 


\section{Введение}

Результаты проводимых в последние десятилетия исследований позволяют считать сердечно-сосудистые заболевания не случайным спутником хронической обструктивной болезни легких, а одним из ее системных проявлений [Кузьмичев и др., 2017; Donaldson et al., 2010; Roversi et al., 2016; Roversi et al., 2014; Trinkmann et al., 2019].

Сердечно-сосудистые заболевания в сочетании с ХОБЛ характеризуются изменением клинической симптоматики, трудностями в диагностике и неблагоприятным прогнозом [Поликутина и др., 2014; Roversi et al., 2014; Westerik et al., 2017]. Несмотря на активный научно-практический интерес к проблеме кардиореспираторной коморбидности, на сегодняшний день остается множество нерешенных вопросов. Не до конца изучены патогенетические звенья развития коронарных событий при ХОБЛ, продолжается поиск ранних и надежных маркеров острых коронарных катастроф у пациентов с ХОБЛ [Наумов и др., 2019; Eapen et al., 2014].

В этом аспекте представляет интерес группа белков теплового шока (HSP), являющихся семейством высококонсервативных специализированных молекул, продуцирующихся при стрессорных воздействиях различного генеза на клетки. HSP функционируют как молекулярные шапероны, тем самым обеспечивая репаративные внутриклеточные процессы [Иванова и др., 2019; Кабалык, 2019; Lichtenauer et al., 2014]. Помимо этого, белки теплового шока во внеклеточном пространстве могут регулировать тонус сосудов [Kirsty et al., 2014; Lichtenauer et al., 2014; Trinkmann et al., 2019] и являться сигналом активации иммунного ответа, включая выработку провоспалительных цитокинов [Calderwood et al., 2016; Guisasola et al., 2015]. Таким образом, роль и значение белков теплового шока многогранно и патогенетически может оказывать как защитное, так и негативное воздействие на органы и системы [Bielecka-Dabrowa, et al., 2009; Trinkmann et al., 2019].

Одним из наиболее изученных представителей семейства белков теплового шока является HSP70. Так, уровень HSP70 в сыворотке ранее признавался потенциальным биомаркером при различных заболеваниях, включая ХОБЛ [Атякшин и др., 2015], инфаркт миокарда. Однако его роль в развитии инфаркта миокарда у коморбидных пациентов с ХОБЛ изучена недостаточно. Исследование данного маркера позволит уточнить механизмы развития сердечно-сосудистых заболеваний у пациентов с ХОБЛ.

Цель: оценить клинико-патогенетическое значение белка теплового шока HSP70 у больных инфарктом миокарда на фоне хронической обструктивной болезни легких.

\section{Объекты и методы исследования}

Было обследовано 65 больных ИМ, из них 28 пациентов имели ИМ в качестве мононозологии, у 37 ИМ развился на фоне ХОБЛ. Группу контроля составили 20 соматически здоровых добровольца, проживающих на территории Астраханской области, сопоставимых с исследуемыми группами по гендерно-возрастным характеристикам.

Обследование больных и наблюдение за группами включенных в исследование проводилось в региональном сосудистом центре ГБУЗ АО Александро-Мариинской областной клинической больницы г. Астрахани в период с 2016 по 2017 гг.

Перед включением в исследование у всех пациентов было получено письменное информированное согласие на участие в данном исследовании.

Группа контроля проходила обследование в поликлиниках г. Астрахани согласно территориальному прикреплению в соответствии с приказом Министерства здравоохранения Российской Федерации от 03.02.2015 № 36 «Об утверждении порядка проведения диспансеризации определенных групп взрослого населения».

Возраст обследованных составил от 45 до 60 лет (средний возраст 52,8 2,4 лет).

Диагноз ИМ устанавливался на основании клинических рекомендаций «Четвертое универсальное определение инфаркта миокарда» Европейского общества кардиологов (2018). Критериями включения в основные группы являлись возраст до 60 лет, II-й тип 
ИМ. В исследование не включались пациенты с другими типами ИМ, с терминальной печеночной и почечной недостаточностью (скорость клубочковой фильтрации менее 30 мл/мин), онкологическими заболеваниями.

В группе больных ИМ на фоне ХОБЛ диагноз легочной патологии, а также тяжесть течения ХОБЛ были верифицированы ранее. Все больные с ХОБЛ имели II-III стадии заболевания вне обострения. Диагноз ХОБЛ устанавливался в соответствии с программой «Глобальная стратегия диагностики, лечения и профилактики хронической обструктивной болезни лёгких» (пересмотр 2019 г.). Медиана длительности ХОБЛ составила $17,5(7,4 ; 24,6)$ лет. Анамнез курения имелся у 100\% пациентов. На момент госпитализации курящими были 87,8\% пациентов с ХОБЛ. Средний индекс курения составил 34,6 пачка-лет. В 96\% случаев наблюдаемыми были мужчины с длительным анамнезом курильщика на момент исследования.

В сыворотке крови определяли концентрацию HSP70 (нг/мл) («Enzo Life Science», США) и уровень ИЛ-6 (пг/мЛ) («ИЛ-6-Вектор-БЕСТ», Россия) иммуноферментным анализом. Для оценки функции внешнего дыхания проведена спирография на аппарате SP-100 «Schiller» (Швейцария). В качестве базового показателя функции внешнего дыхания оценивался объем форсированного выдоха в первую секунду $\left(\mathrm{O}_{1}\right)$. Исследование газового состава крови проводились на анализаторе газов крови ABL800 FLEX (Россия).

Статистическая обработка полученных результатов осуществлялась с использованием пакета прикладных программ Statistica 11,0 (StatSoft, Inc.). В связи с тем, что признаки в исследуемых группах имели распределение, отличное от нормального, для каждого показателя вычисляли медиану и интерпроцентильные размахи (25-й и 75-й процентили). Достоверность различий определялась с помощью критерия Манна - Уитни. Наличие и силу связи между изучаемыми показателями оценивали с помощью непараметрического коэффициента ранговой корреляции Спирмена (R). Отличия считались статистически значимыми при р $<0,05$.

\section{Результаты и их обсуждение}

При исследовании уровней двух биомаркеров: HSP70 и IL-6, установлено, что у больных с коморбидной патологией оба эти показателя были достоверно выше не только с группой здоровых лиц, но и с группой с инфарктом миокарда (табл.1).

Таблица 1

Table 1

Уровни HSP70 и IL-6 у больных и здоровых лиц

The level of HSP70 and IL-6in patients and healthy

\begin{tabular}{|l|c|c|}
\hline \multirow{2}{*}{ Группы наблюдения } & \multicolumn{2}{|c|}{ Показатели } \\
\cline { 2 - 3 } & HSP70 (нг/мл) & IL-6 (пг/мл) \\
\hline Больные ИМ $(\mathrm{n}=28)$ & $0,598[0,09-0,46]^{* *}$ & $8,6[6,1-19,9]^{*}$ \\
\hline Больные ИМ + ХОБЛ $(\mathrm{n}=37)$ & $0,954[0,29-0,56]^{* *}$ o & $15,6[6,5-28,4]^{* *}$ o \\
\hline Группа контроля $(\mathrm{n}=20)$ & $0,12[0,05 ; 0,37]$ & $0,97[0,2-4,2]$ \\
\hline
\end{tabular}

Примечание: * - $<0,05$ - статистически достоверные различия по сравнению с группой контроля (в скобках 25-й и 75-й процентили); $* *-p<0,01-$ статистически достоверные различия по сравнению с группой контроля; ${ }^{\circ}-\mathrm{p}<0,05$ - статистическая достоверность уровня HSP70 и IL-6 в группе больных ИМ на фоне ХОБЛ по сравнению с группой больных ИМ.

При анализе уровня HSP70 в зависимости от времени поступления пациентов в стационар установлено, что у больных ИМ, поступивших в первые 12 часов от момента развития клиники ИМ, уровень HSP70 был достоверно выше по сравнению как с группой контроля $(\mathrm{p}<0,01)$, так и с группой больных ИМ, поступивших позже 12 часов от момента развития клинической симптоматики (р<0,05) (табл. 2). 
Уровень HSP70 у больных ИМ в зависимости от времени госпитализации, нг/мл

The level of HSP70 in MI patients depending on the time of hospitalization, $\mathrm{ng} / \mathrm{ml}$

\begin{tabular}{|l|c|c|}
\hline \multirow{2}{*}{ Группы наблюдения } & \multicolumn{2}{|c|}{ Сроки госпитализации } \\
\cline { 2 - 3 } & До 12 часов & Позже 12 часов \\
\hline Больные ИМ $(\mathrm{n}=28)$ & $0,43[0,29 ; 0,56]^{*}$ & $0,30[0,08 ; 0,41]$ \\
\hline Больные ИМ + ХОБЛ $(\mathrm{n}=37)$ & $1,05[0,28 ; 0,93]^{* *}$ o & $0,48[0,05 ; 0,37]$ \\
\hline
\end{tabular}

Примечание: * $-\mathrm{p}<0,05, * *-\mathrm{p}<0,01-$ статистически достоверные различия уровня HSP70 в группе больных между собой; ИМ на фоне ХОБЛ по сравнению с группой больных ИМ, o - p<0,05 - статистическая достоверность уровня HSP70 в группе больных ИМ на фоне ХОБЛ по сравнению с всеми группами больных (в скобках 25-й и 75-й процентили).

Самый высокий уровень HSP70 - 1,05 $(0,28 ; 0,93)$ нг/мл отмечался в группе больных ИМ на фоне ХОБЛ, поступивших в стационар в ранние сроки от момента развития ИМ, что было статистически значимо выше, чем в группе контроля ( $<<0,01)$, группе больных ИМ, поступивших в аналогичные сроки $(\mathrm{p}<0,05)$ и в группе больных ИМ на фоне ХОБЛ, поступивших позже 12 часов от момента развития клинических проявлений ИМ $(\mathrm{p}<0,05)$.

Далее была проанализирована зависимость уровня HSP70 от глубины поражения миокарда в группах больных как с мононозологией, так и с коморбидной патологией, поступивших в ранние сроки развития ИМ (табл. 3).

Таблица 3

Table 3

Уровень HSP70 в зависимости от глубины поражения миокарда, нг/мл

The level of HSP70 depending on the depth of myocardial lesion, $\mathrm{ng} / \mathrm{ml}$

\begin{tabular}{|l|c|c|}
\hline Глубина поражения & ИМ $(\mathrm{n}=28)$ & ИМ + ХОБЛ $(\mathrm{n}=37)$ \\
\hline Не трансмуральное & $0,27[0,07-0,38]$ & $0,531[0,15-0,46]$ \\
\hline Трансмуральное & $0,75[0,09-0,48]^{\circ}$ & $1,31[0,28-0,79]^{\circ *}$ \\
\hline
\end{tabular}

Примечание: * - p $<0,05$ - статистическая достоверность уровня HSP70 в группе больных ИМ на фоне ХОБЛ по сравнению с группой больных ИМ при соответствующей глубине поражения; ${ }^{\circ}-\mathrm{p}<0,05$ - статистическая достоверность уровня HSP70 в группе больных с трансмуральным поражением по сравнению с группой больных с не трансмуральным поражением (в скобках 25-й и 75-й процентили).

При трансмуральном поражении миокарда самый высокий уровень HSP70 отмечался у больных ИМ на фоне ХОБЛ, что было статистически значимо выше по сравнению как с группой больных ИМ с трансмуральным поражением $(\mathrm{p}<0,05)$, так и с группой коморбидных больных с не трансмуральным поражением миокарда $(\mathrm{p}<0,05)$.

При проведении корреляционного анализа между уровнем HSP70 и рядом лабораторно-инструментальных показателей установлено, что у больных ИМ на фоне ХОБЛ отмечается прямая корреляционная связь с объемом форсированного выдоха в первую секунду $\left(\mathrm{O} Ф \mathrm{~B}_{1}\right)(\mathrm{R}=0,34, \mathrm{p}<0,05)$ (табл. 4).

Также уровень HSP70 находился в прямой корреляционой зависимости от парциального давления кислорода в крови $\left(\mathrm{pO}_{2}\right)(\mathrm{R}=0,65, \mathrm{p}<0,05)$ и обратной корреляционой зависимости от парциального давления углекислого газа в крови $\left(\mathrm{pCO}_{2}\right)(\mathrm{R}=-0,38, \mathrm{p}<0,05)$.

Учитывая, что появление внеклеточного Hsp70, играющего двойную роль как шаперона и цитокина, является сигналом активации иммунного ответа с индукцией провоспалительных цитокинов, таких как, например, IL-6 [14], мы проанализировали корреляционную зависимость между этими двумя параметрами. Была установлена прямая корреляционная связь уровня HSP70 с уровнем интерлейкина-6 (IL-6) $(\mathrm{R}=0,83, \mathrm{p}<0,05)$. 
Корреляция между уровнем HSP70 у больных ИМ на фоне ХОБЛ и рядом лабораторно-инструментальных показателей

Correlation between the level of HSP70 in MI patients with COPD and a number of laboratory and instrumental indicators

\begin{tabular}{|l|c|c|}
\hline \multicolumn{1}{|c|}{ Показатели } & Коэффициент корреляции, $\mathrm{R}$ & Достоверность \\
\hline $\mathrm{OФB}_{1}$ & 0,34 & $\mathrm{P}<0,05$ \\
\hline $\mathrm{IL}-6$ & 0,83 & $\mathrm{P}<0,05$ \\
\hline $\mathrm{PO}_{2}$ & 0,65 & $\mathrm{P}<0,05$ \\
\hline $\mathrm{PCO}_{2}$ & $-0,38$ & $\mathrm{P}<0,05$ \\
\hline
\end{tabular}

\section{Обсуждение результатов}

Наибольший уровень HSP70 выявлен в коморбидной группе. Учитывая тот факт, что ХОБЛ - системное, стрессовое, воспалительное и хроническое заболевание, можно предположить, что некротические клетки легких или мононуклеарные клетки периферической крови могут являться источником повышенного содержания HSP70. С одной стороны, увеличение содержания HSP70 создает механизм адаптации защитных сил клетки, а с другой - избыточное его накопление может сопровождаться экспрессией белков теплового шока и, как следствие, выбросом их в кровь и активацией цитокинового каскада, хорошим индикатором которого является IL-6. HSP70 может активировать иммунную систему и, таким образом, участвовать в местных воспалительных реакциях, подвергая клетки миокарда длительное время стрессовому состоянию, что способствует накоплению в этих клетках повреждающих белков с последующим увеличением синтеза шаперонов.

У больных ИМ выявлено существенное повышение уровня HSP70 по сравнению с лицами контрольной группы, вне зависимости от сроков госпитализации. Эти данные согласуются с результатами, полученными Zhang X с соавторами в 2010 году. В проведенном авторами исследовании уровень HSP70 у пациентов с ишемической болезнью сердца был статистически выше, чем в контроле, с максимальными значениями у больных с острым коронарным синдромом [Zhang et al., 2010]. Аналогичные данные были получены Dybdahl с соавторами в 2005 году, выявившими повышенные концентрации HSP70 в сыворотке крови у пациентов с ИМ по сравнению с больными стенокардией [Dybdahl et al., 2005]. По-видимому, гиперпродукция HSP70 в данном случае имеет стресс-протективный характер относительно периинфарктной зоны ишемизированного миокарда. Более высокие значения HSP70 у пациентов с трансмуральным поражением миокарда можно объяснить большей массой кардиомиоцитов, подверженных ишемическому стрессу.

В группе больных ИМ на фоне ХОБЛ уровень HSP70 максимален в ранние сроки от момента развития ИМ. Можно предположить, что перманентная гипоксия, обусловленная ИМ и ХОБЛ, усугубляет стрессорное воздействие на кардиомиоциты. Полученные данные позволяют говорить о HSP70 как маркере ранней диагностики ИМ у пациентов с ХОБЛ, а взаимосвязь с глубиной поражения миокарда наводит на мысль о возможности использования уровня HSP70 в качестве маркера тяжести течения ИМ у коморбидных пациентов с ХОБЛ.

Прямая корреляционная взаимосвязь с уровнем ИЛ-6, обнаруженная в нашем исследовании, подтверждает данные, полученные Dybdahly (2005). В своем исследовании авторы обнаружили прямую взаимосвязь между уровнями HSP70 у больных ИМ с уровнем ИЛ-6, КФК-МВ и тропонином [Dybdahl et al., 2005]. Такая связь подтверждает инициацию продукции HSP70 под воздействием воспалительного каскада - важного патогенетического звена в развитии сосудистых катастроф. В то же время Haider (2019) не было обнаружено корреляции между уровнями HSP70 и стандартными лабораторными показателями у больных с обширной травмой [Haider et al., 2019]. 
Наличие прямой корреляционной связи между уровнем HSP70 и величиной ОФВ 1 свидетельствует об угнетающем влиянии обструктивных нарушений на процессы репарации, в том числе и в миокарде, и подчеркивает негативное влияние ХОБЛ на развитие и течение коронарной симптоматики у больных с ХОБЛ. Это утверждение подкрепляется и наличием корреляционных связей между газами крови и HSP70.

\section{Вывод}

Результаты проведенного исследования свидетельствуют, что HSP70 может использоваться в качестве маркера тяжести течения ИМ у больных ИМ на фоне ХОБЛ в ранние сроки от момента развития заболевания. Это будет способствовать раннему выявлению коронарных событий у больных с ХОБЛ, что особенно важно с учетом высокой частоты стертых форм ИМ и атипичной симптоматики ИМ у больных ХОБЛ.

\section{Список литературы}

1. Атякшин Д.А., Цветикова Л.Н., Лобеева Н.В., Будневский А.В., Овсянников Е.С. 2015. Показатели иммунного статуса при хронической обструктивной болезни легких. Успехи современного естествознания. 9: 195-197.

2. Иванова Н.М., Цыбиков Н.Н., Сормолотова И.Н., Цыбиков Н.Б., Иванов М.О. 2019. Шапероны как связующее звено между атопическим дерматитом и аллергическим ринитом. Медицинский вестник Северного Кавказа. 14 (2): 349-351.

3. Кабалык М.А. 2019. Клинико-патогенетическое значение белков теплового шока с массой 70 и 27 кДв при остеоартрите. Научно-практическая ревматология. 55 (2): 187-191.

4. Кузьмичев Б.Ю., Полунина Е.А., Кузьмичев К.Ю., Липницкая Е.А., Аджян М.С. 2017. Исследование уровня гомоцистеина у пацинтов с инфарктом миокарда на фоне хронической обструктивной болезни легких. Астраханский медицинский журнал. 12 (4): 44-50.

5. Наумов А.В., Сароянц Л.В., Прокофьева Т.В., Полунина О.С., Полунина Е.А. 2019. Информативность цитокинового профиля в прогнозировании осложнений у больных инфарктом миокарда на фоне хронической обструктивной болезни легких. Медицинский алфавит. 22 (397): 33-36.

6. Поликутина О.М., Слепынина Ю.С., Баздырев Е.Д., Каретникова В.Н., Барбараш О.Л. 2014. Исходы инфаркта миокарда с подъемом сегмента ST у пациентов с хронической обструктивной болезнью легких в Кемеровской области. Российский Кардиологический Журнал. 7 (111): 91-97.

7. Bielecka-Dabrowa A., Barylski M., Mikhailidis D.P., Rysz J., Banach M. 2009. HSP 70 and atherosclerosis protector - or activator? Expert Opinion on Therapeutic Targets. 13: 307-317.

8. Calderwood S.K., Gong J. Murshid A. 2016. Extracellular HSPs: The Complicated Roles of Extracellular HSPs in Immunity. Front. Immunol. 7: 159.

9. Donaldson G.C., Hurst J.R., Smith C.J., Hubbard R.B., Wedzicha J.A. 2010. Increased risk of myocardial infarction and stroke following exacerbation of COPD. Chest. 137: 1091-1097.

10. Dybdahl B., Slørdahl S.A., Waage A., Kierulf P., Espevik T., Sundan A. 2005. Myocardial ischemia and the inflammatory response: release of heat shock protein 70 after myocardial infarction. Heart. 91: 299-304.

11. Eapen D.J., Ghasemzadeh N., MacNamara J.P., Quyyumi A. 2014. The Evaluation of Novel Biomarkers and the Multiple Biomarker Approach in the Prediction of Cardiovascular Disease. Curr. Cardiovasc. Risk. Rep. 8: 408.

12. Guisasola M.C., Ortiz A., Chana F., Alonso B. Vaquero J. 2015. Early inflammatory response in polytraumatized patients: Cytokines and heat shock proteins. A pilot study. Orthop. Traumatol. Surg. Res. 101: 607-611.

13. Haider Th., Simader E., Glück O., Ankersmit H.J., Heinz Th., Hajdu S., Negrin L.L. 2019. Systemic release of heat-shock protein 27 and 70 following severe trauma. Scientific Reports. 9: 9595.

14. Kirsty M. Danielson1 and Saumya 2014. Das Extracellular Vesicles in Heart Disease: Excitement for the Future Exosomes Microvesicles. 2: 1. doi: 10.5772 
15. Lichtenauer M., Zimmermann M., Nickl S., Lauten A., Goebel B., Pistulli R., Yilmaz A., Figulla H.R., Ankersmit H.J., Jung C. 2014. Transient hypoxia leads to increased serum levels of heat shock protein-27, -70 and caspase-cleaved cytokeratin 18. Clin. Lab. 6: 323-328.

16. Roversi S., Fabbri L.M., Sin D.D., Hawkins N.M., Agustí A. Chronic obstructive pulmonary disease and cardiac diseases. 2016. An urgent need for integrated care. Am. J. Respir. Crit. Care. Med. 194: 1319-1336.

17. Roversi S., Roversi P., Spadafora G., Rossi R., Fabbri L.M. 2014. Coronary artery disease concomitant with chronic obstructive pulmonary disease. Eur. J. Clin. Invest. 44: 93-102.

18. Saban K.L., Hoppensteadt D., Bryant F.B., Devon H.A. 2013. Social determinants and heat shock protein-70 among african american and non-hispanic white women with atherosclerosis: a pilot study. Biol. Res. Nurs. 16 (3): 258-265.

19. Trinkmann F., Saur J., Borggrefe M. and Ibrahim Aki J. 2019. Cardiovascular Comorbidities in Chronic Obstructive Pulmonary Disease (COPD) - Current Considerations for Clinical Practice Clin. Med. 8 (1): 69.

20. Westerik J.A., Metting E.I.,van Boven J.F., Tiersma W., Kocks J.W., Schermer T.R. 2017. Associations between chronic comorbidity and exacerbation risk in primary care patients with COPD. Respir. Res. 18: 31.

21. Zhang X., Xu Z., Zhou L., Chen Y., He M., Cheng L., Hu F.B., Tanguay R.M., Wu T. 2010. Plasma levels of Hsp70 and anti-Hsp70 antibody predict risk of acute coronary syndrome. Cell. Stress. Chaperones. 15: 675-686.

\section{References}

1. Atyakshin D.A., Tsvetikova L.N., Lobeeva N.V., Budnevskiy A.V., Ovsyannikov E.S. 2015. Pokazateli immunnogo statusa pri khronicheskoy obstruktivnoy bolezni legkikh [Indicators of the immune status in chronic obstructive pulmonary disease]. Uspekhi sovremennogo estestvoznaniya. 9: 195-197.

2. Ivanova N.M., Tsybikov N.N., Sormolotova I.N., Tsybikov N.B., Ivanov M.O. 2019. Shaperony kak svyazuyushchee zveno mezhdu atopicheskim dermatitom i allergicheskim rinitom [Chaperones as a link between atopic dermatitis and allergic rhinitis]. Meditsinskiy vestnik Severnogo Kavkaza. 14 (2): 349-351.

3. Kabalyk M.A. 2019. Kliniko-patogeneticheskoe znachenie belkov teplovogo shoka s massoy 70 i $27 \mathrm{kDv}$ pri osteoartrite [Clinical and pathogenetic value of heat shock proteins with a mass of 70 and $27 \mathrm{KdV}$ in osteoarthritis]. Nauchno-prakticheskaya revmatologiya. 55 (2): 187-191.

4. Kuz'michev B.Yu., Polunina E.A., Kuz'michev K.Yu., Lipnitskaya E.A., Adzhyan M.S. 2017. Issledovanie urovnya gomotsisteina u patsintov s infarktom miokarda na fone khronicheskoy obstruktivnoy bolezni legkikh [Study of the level of homocysteine in patients with myocardial infarction against the background of chronic obstructive pulmonary disease]. Astrakhanskiy meditsinskiy zhurnal. 12 (4): 44-50.

5. Naumov A.V., Saroyants L.V., Prokof'eva T.V., Polunina O.S., Polunina E.A. 2019. Informativnost' tsitokinovogo profilya $\mathrm{v}$ prognozirovanii oslozhneniy u bol'nykh infarktom miokarda na fone khronicheskoy obstruktivnoy bolezni legkikh [Informative cytokine profile in predicting complications in patients with myocardial infarction against the background of chronic obstructive pulmonary disease]. Meditsinskiy alfavit. 22 (397): 33-36.

6. Polikutina O.M., Slepynina Yu.S., Bazdyrev E.D., Karetnikova V.N., Barbarash O.L. 2014. Iskhody infarkta miokarda s pod"emom segmenta ST u patsientov s khronicheskoy obstruktivnoy bolezn'yu legkikh v Kemerovskoy oblasti [Outcomes of ST-segment elevation myocardial infarction in patients with chronic obstructive pulmonary disease in the Kemerovo region]. Rossiyskiy Kardiologicheskiy Zhurnal. 7 (111): 91-97.

7. Bielecka-Dabrowa A., Barylski M., Mikhailidis D.P., Rysz J., Banach M. 2009. HSP 70 and atherosclerosis protector - or activator? Expert Opinion on Therapeutic Targets. 13: 307-317.

8. Calderwood S.K., Gong J. Murshid A. 2016. Extracellular HSPs: The Complicated Roles of Extracellular HSPs in Immunity. Front. Immunol. 7: 159.

9. Donaldson G.C., Hurst J.R., Smith C.J., Hubbard R.B., Wedzicha J.A. 2010. Increased risk of myocardial infarction and stroke following exacerbation of COPD. Chest. 137: 1091-1097. 
10. Dybdahl B., Slørdahl S.A., Waage A., Kierulf P., Espevik T., Sundan A. 2005. Myocardial ischaemia and the inflammatory response: release of heat shock protein 70 after myocardial infarction. Heart. 91: 299-304.

11. Eapen D.J., Ghasemzadeh N., MacNamara J.P., Quyyumi A. 2014. The Evaluation of Novel Biomarkers and the Multiple Biomarker Approach in the Prediction of Cardiovascular Disease. Curr. Cardiovasc. Risk. Rep. 8: 408.

12. Guisasola M.C., Ortiz A., Chana F., Alonso B. Vaquero J. 2015. Early inflammatory response in polytraumatized patients: Cytokines and heat shock proteins. A pilot study. Orthop. Traumatol. Surg. Res. 101: 607-611.

13. Haider Th., Simader E., Glück O., Ankersmit H.J., Heinz Th., Hajdu S., Negrin L.L. 2019. Systemic release of heat-shock protein 27 and 70 following severe trauma. Scientific Reports. 9: 9595.

14. Kirsty M. Danielson1 and Saumya 2014. Das Extracellular Vesicles in Heart Disease: Excitement for the Future Exosomes Microvesicles. 2: 1. doi: 10.5772

15. Lichtenauer M., Zimmermann M., Nickl S., Lauten A., Goebel B., Pistulli R., Yilmaz A., Figulla H.R., Ankersmit H.J., Jung C. 2014. Transient hypoxia leads to increased serum levels of heat shock protein-27, -70 and caspase-cleaved cytokeratin 18. Clin. Lab. 6: 323-328.

16. Roversi S., Fabbri L.M., Sin D.D., Hawkins N.M., Agustí A. Chronic obstructive pulmonary disease and cardiac diseases. 2016. An urgent need for integrated care. Am. J. Respir. Crit. Care. Med. 194: 1319-1336.

17. Roversi S., Roversi P., Spadafora G., Rossi R., Fabbri L.M. 2014. Coronary artery disease concomitant with chronic obstructive pulmonary disease. Eur. J. Clin. Invest. 44: 93-102.

18. Saban K.L., Hoppensteadt D., Bryant F.B., Devon H.A. 2013. Social determinants and heat shock protein-70 among african american and non-hispanic white women with atherosclerosis: a pilot study. Biol Res Nurs. 16(3): 258-265.

19. Trinkmann F., Saur J., Borggrefe M. and Ibrahim Aki J. 2019. Cardiovascular Comorbidities in Chronic Obstructive Pulmonary Disease (COPD) - Current Considerations for Clinical Practice Clin. Med. 8(1): 69.

20. Westerik J.A., Metting E.I.,van Boven J.F., Tiersma W., Kocks J.W., Schermer T.R. 2017. Associations between chronic comorbidity and exacerbation risk in primary care patients with COPD. Respir. Res. 18: 31.

21. Zhang X., Xu Z., Zhou L., Chen Y., He M., Cheng L., Hu F.B., Tanguay R.M., Wu T. (2010) Plasma levels of Hsp70 and anti-Hsp70 antibody predict risk of acute coronary syndrome. Cell Stress Chaperones 15: 675-686.

\section{Ссылка для цитирования статьи For citation}

Наумов А.В., Прокофьева Т.В., Полунина О.С., Сароянц Л.В. 2020. Клиникопатогенетическое значение белка теплового шока HSP70 при инфаркте миокарда на фоне хронической обструктивной болезни легких. Актуальные проблемы медицины, 43(1): 38-45. DOI

Naumov A.V., Prokofieva T.V., Polunina O.S., Saroyants L.V. 2020. Clinical and pathogenetic application of the HSP70 heat shock protein in myocardial infarction on the background of chronic obstructive lung disease. Challenges in Modern Medicine, 43(1): 38-45 (in Russian). DOI 